\title{
Value Co-Creation Mechanisms in a Born Digital Short-form Video Platform: Kuaishou
}

\author{
William W. Baber \\ Kyoto University \\ baber@gsm.kyoto-u.ac.jp
}

\author{
Chen Yao \\ yc19941119@gmail.com
}

\begin{abstract}
This paper focuses on the value co-creation mechanism of the value constellation of a digital services platform exemplified by the case of Kuaishou. This born-digital platform business is among the largest short-form video platforms in the world connecting makers, shoppers, performers, advertisers, and others. Using business model theory to understand the firm, this study employs "gravities theory" as the main framework to identify where and how value is created and shared among the platform's participants. The study concludes that valuerecognizing gravity, value-sharing gravity, and value realizing-gravity are found in the Kuaishou value constellation. Kuaishou forms the center dominating the internal value gravities while value gravities are found also externally, around the platform. Analysis of the business model shows that the platform's distinct culture is a valuable mechanism of success within China, but may be a contributing factor to the so far slow international development.
\end{abstract}

\section{Introduction}

As business activities become more complex, especially with the rise of internet-based industries, customers are becoming more involved in the value creation process [1]. The strategic focus of enterprises has also changed from creating and delivering value to providing customers with a platform for value creation [2]. In this complex value network, the role of enterprises and how to participate in value creation are a focus of researchers' attention [3]. As a relatively new concept, value co-creation provides an effective perspective for such research.

In China, short-form video has emerged as the most popular way to socialize and entertain. Compared to traditional graphic expression, short videos can deliver rich visual and auditory information quickly, while meeting users' needs for fast-paced information. The two largest such platforms, TikTok and Kuaishou, both originate in China, and no major competitors have appeared elsewhere, making this phenomenon of particular interest to non-Chinese academics and practitioners interested in the business models embedded within these platform ecosystems. Kuaishou, in February 2021, made its initial public offering raising immense sums and attracting attention worldwide. The firm was born a digital organization, i.e. it never had a tangible non-digital product [4]. Growth in China has been fast for Kuaishou, but less quick internationally. Platform users include content creators and their audiences as well as small makers of all sorts. Enabling users to spontaneously create value on the platform and playing the role of the platform itself in this complex value network is also central to the short-form video platform strategy.

As yet the interaction, attraction, and reinforcement of participants and their value cocreation in a multisided platform remains unclear. Network effects have been elucidated in part, however the relationships of peripheral participants to the platform and the relationships among peripheral participants remain incompletely understood. This study seeks to examine the intimate interactions of participants within platforms [5] to learn how the participants in a multisided platform function as members of the value constellation by modeling the business and its logics [6].

\section{Literature review}

In the traditional industrial view, customers destroy the value created by the producers. "For producers, industrial value was 'realized' in the transaction which joined and separated them from customers" [7], pg. 49. However, due to sociotechnical breakthroughs, an alternative view arose that customers also create value as coproducers [7]. As service-dominant logic was introduced, value co-creation theory brought a new perspective to value creation study. In servicedominant logic, the enterprise only provides value 
proposition by offering resources and co-creates value with the customer. It is the customer who determines the value of the service [2]. Although Vargo and Lusch [2] noticed the importance of other social and economic actors who join in the valuecreation process by resource integration and allocation, study still put focus on the interaction of the enterprise and the customer. Based on servicedominant logic, a service ecosystem theory regarding a network of relationships appeared [8]. Under a service ecosystem framework, "all actors are both providers and beneficiaries, and the "producers" and "consumers" distinction vanishes" [8], pg. 148. Therefore, the producer-consumer relationship expanded to be seen as "actor-to-actor", which indicates a comprehensive value-creation network of relationships among all social and economic actors connected with resource integration and mutual service provision. This paper considers digital ecosystems to include dynamic interaction among autonomous [3] participants as individuals or categories that symbiotically [9] create niches [10] as they divide labor among themselves [9] though dependent on a hub business or platform [11].

As the Internet enables development of value creating networks, case studies have appeared on value creation mechanisms in internet-based business. Yang and Tao [12] found that the social value co-creation mode of Xiaomi corporation changes from a physical value chain and value matrix to a flexible value network, which consists of user linked value co-creation, user interaction value cocreation, and re-construction value co-creation. $\mathrm{Li}$, Chen and Zhang [13] studied the third party billing model of Mafengwo, a travel information platform in China, from service-dominant logic perspective, and found that the platform, user, and third party cocreate value through different paths based on different drivers. Wang and Sun [14] studied business model innovation in Tiktok from a value creation perspective, separating value proposition, value cocreation, value acquisition and, value realization. Live-streaming in entertainment has been considered in the context of Kuaishou, though with emphasis on entertainment and state managed governance [15], [16]. Such firms usually from inception have mainly digital offerings exploiting digitalized value chains and are identified as Born Digital [17]. Business models help to describe and visualize organizations, allowing identification of advantages, disadvantages, opportunities, risks, profitability, and so on including in Information Systems firms [18]. The method of modeling resources, network, revenue, and value creation involves consideration of especially qualitative information about processes and relationships [18]. Further, business models help identify other players in the value constellation and their roles by visualizing relationships among them.

We turn our attention now to the value constellation, the actors within and around the central firm [7], [19]. The value chain model proposed by Michael Porter [20] proposes that the key point of corporate strategy is to determine the position of the company on the value chain and add value to the product with proper activities. This model leads to a common understanding that value creation processes are sequential and linear. But with the change of technology and environment, value chain theory is no longer able to explain the complicated value creation process involved with different players [7].

Accordingly, Ramírez [7] mentioned that within a value constellation, a single business cannot provide everything, so the main task of business is to conceive and maintain value-creating systems by reconfiguring its relationships and business systems. Thus, business creates value by co-producing offerings that mobilize customers to create value of their own. Successful businesses do not just add value, but they also reinvent value. Vargo et al. [8] said that all actors (economic and social) should be understood in terms of a common set of generic functions - resource integration and mutual service provision - that create value-creation networks. Luo [1] furthered the theoretical explanations around value constellation in digital firms describing it as a gravity aggregation of value creation formed by manufacturers, suppliers, partners, distributors, and customers. The gravity can be divided into (1) valuerecognizing gravity: value concept that is accepted and recognized by all actors; (2) value-achieving gravity: value can be realized by appropriate mechanisms; (3) value-sharing gravity: every value chain of a value constellation contributes to an overall value sharing system involving all actors. Within the value constellation, the focus of enterprise changes from creating value for customers to cocreating value together with customers. In order to provide attractive product/service, the enterprise is required to co-create value with customers, suppliers, allies and other actors, thus, the strategy focus is to reconfigure different relationships and business systems as well as to build or join the value constellation. The competitive advantage of the enterprise comes from the ability to create valuecreating systems.

When considering the value creation process in a value network consisting of third parties, users, content creators, and content platforms, this "three forces" theory provides a way to broadly understand the mechanism of creating value jointly. These forces 
are referred to as gravities, in line with Luo's solar system analogy and emphasizing the attraction among the players [1]. After applying Luo's theory to the value constellation of content platforms, it is found that content creators and viewers are attracted by the value-recognizing gravity generated by a content platform; viewers and third parties are attracted by the value sharing gravity arising from a content platform; content creators and third parties are attracted by the value-realizing gravity provided by a content-platform.

Content creators and viewers are attracted by the content platform because they may not only comment on the concept and ideas that are recognized by both parties, but also find virtual communities with similar interest to join as they build trusting relationships with each other. At they same time they may express and develop their identities while making value recreationally [21]. Third parties and viewers can share the value that they created through the content platform, such as products, experiences, ideas, and even feedback. As for content creators and the third parties, they are attracted by the value realizing function of the content platform.

\section{Methodology}

This study examines a firm, Beijing Kuaishou Technology, to understand the business model and the workings of the value constellation of its platform, Kuaishou. The platform is remarkable for its growth as well as its empowerment of small-scale entrepreneurs and firms in less affluent cities. Additionally, its casual, accessible "on the level" marketing and culture made it of interest to the authors. An illustrative case study, relevant data was collected from public sources, including the firm's websites, reports, and app rather than from interviews. Academic literature about Kuaishou and similar firms was reviewed as were other sources of data including news media and government statistics. The assembled data was condensed and ordered [22] through construction of business models and assessment of the value constellation.

Business model analysis helps to evaluate the Kuaishou ecosystem by understanding interactions within the value constellation. The business models identified and mapped for this study reveal relationships, movement of resources, and transitions as value moves from creation to realization.

This study also analyzes the case firm with the value gravities framework proposed by Luo (2006). Luo theorizes that value constellations draw participants into particular areas of creating, realizing, and sharing value; it is their aggregate activity that makes the overall system function. At the center of such a constellation is a focal firm, in this study, the video sharing and ecommerce platform Kuaishou. This approach places the focal firm in the metaphorical position of the sun and observes the movement of partners, customers, and competitors around that sun as well as their impact on each other. Rather than seeing discrete value chains, this approach considers aggregate value chains as gravities in the total system.

\section{The Case: Kuaishou}

Kuaishou is one of the top two social video apps in China, originally appearing in 2011 as a GIF editing tool for mobile, a digital product which appeared on other platforms. In 2012, Kuaishou released into a short video app for social sharing and launched its platform in 2013, gaining the position of largest streaming platform worldwide by revenue in 2017 [23]. In 2019, Kuaishou became the $2^{\text {nd }}$ largest live-streaming e-commerce platform in the world [23]. Despite weak performance post-IPO, the platform achieved 1 billion monthly active users in June 2021 [24]. Kuaishou, unlike its larger competitor TikTok, dominates market share in lesser cities across China and has made this position a virtue, fixing it in the platform's culture [15]. That market, and giving voice and opportunity to its members, is a goal emphasized by the founder in interviews [25]. The firm has from inception had a focus on producing benefits for those lesser cities and rural areas in China that have been somewhat left behind, aiming to include people from the countryside and lesser cities. This mission to China is locked into the firm's thinking and the app's functions [15] and is reflected in the founders' stated mission to give voice and income to overlooked China [26]. In February 2021, Kuaishou became a publicly listed firm with a total approximate evaluation of $\$ 159$ billion upon its market debut [27], making billionaires of its founders, Su Hua and Cheng Yixiao. International versions of the app appeared even in 2017 in Korea, Russia, Thailand, and Indonesia [28], though with little success. Advances were made when the app briefly topped the download rankings in several countries including USA 2020 [29] before being blocked due to sharing issues [30]. Other international paths have also been bumpy as the app was banned in India in 2020, where it is known as Snack Video, for political concerns. With an all-digital history but slow expansion into international markets making little contribution to corporate revenues despite success at home, Kuaishou falls into the taxonomy of Born Digital, 
Gradually Global [17]. Meanwhile, its closest competitor, TikTok, is seen as Born Digital/Global due to its early international successes. Why then, with evidence of international intent, has Kuaishou remained "gradual"? Examination of the business model, the members of its ecosystem, and their value co-creating relationships helps to clarify.

\subsection{Business model of Kuaishou}

Traditional content platforms including shortform video platforms generate revenue from advertisements as well as from money from virtual gifts of appreciation, sometimes referred to as tips, by users. As for advertisement fees, marketing companies contact a multiple channel network (MCN) to get short videos made by its content makers based on advertiser requests. Such short videos are released either by the content makers or directly by the platform eventually to contribute to the revenue of the platform. Additionally, users can reward the content they like with small amounts of money. These rewards are in the form of electronic stickers that function as de facto stamps of approval or recommendations. The e-stickers may appear as icons of beer mugs, turkey legs or as grander images like dragons. The sender pays a set fee for the esticker and the content creator shares the fee with the Kuaishou platform, approximately half and half. This is an important source of income for short-form video platforms, but in the case of Kuaishou, it represents fully two thirds of 2020 revenue [27].

Since Taobao's combining of live-streaming with sales promotion, this way of selling things has been adopted by other e-commerce platforms and short-form video platforms including Kuaishou. Product suppliers contact MCNs to find suitable livestreamers as sales representatives. In a live-streaming video, viewers can place an order while watching the product information illustrated by a live-streamer. If the viewer likes the product, he/she can click the icon on the screen and the shopping page will pop up. Those shopping pages are mainly e-commerce platforms such as Taobao and JD, supported by quick payment systems such as Alipay and WeChat. The entire shopping process considers the shopping habits of various users, using a variety of internet platforms and fast page jumping, so that users can quickly complete their shopping without additional logins. MCNs of this sort, including Kuaishou, bring together not merely buyers and sellers, but also other members of its business ecosystem such as intermediaries that provide services [9] and the modules [31] that comprise platform architecture.
We can look at the flow of money in the Kuaishou value constellation to understand the sustainability of the entire ecosystem. Advertisement fees and membership fees are revenue for Kuaishou as well as other content platforms. Revenue of livestreaming can be divided into two parts: (1) e-stickers showing appreciation for live-streaming. Kuaishou charges $50 \%$ of these received from live-streaming viewers and leaves $50 \%$ to the content creators and live-streamers; (2) online shopping payment. During live-streaming, users can place an order via the shopping page of an e-commerce platform such as Taobao as well as through the e-commerce function embedded in the Kuaishou app; the shopping fees associated with the order revert to Kuaishou.

After receiving order information from the ecommerce platform, product suppliers dispatch product as soon as possible and get paid via a third party payment app minus the service fee. Sales representatives and content creators/live-streamers receive $50 \%$ of the appreciation gifts as well commissions from product suppliers based on the quantity of the products sold or specific amounts of money as per their agreement. The revenue movement is presented in Figure 1 below.

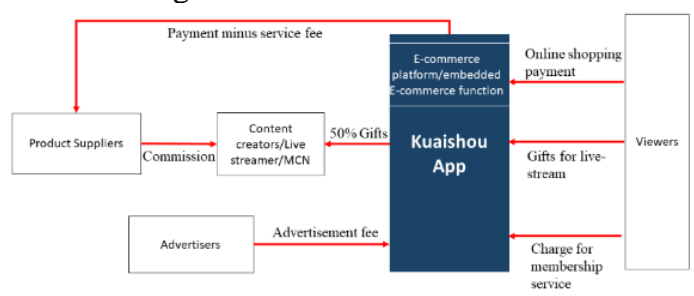

Figure 1. Revenue Model of Kuaishou

According to the above information on Kuaishou and its value co-creators, we can conceive a simplified business model based on the value that each player provides. Basically, all the interactions can be divided into three value co-creation relationships. (1) Kuaishou app and content creator/live streamer. In this value co-creation relationship, Kuaishou app provides with the platform for short video and live-streaming with a variety of services and functions. Kuaishou also rewards the content creators/live streamers to encourage their content creation, a key resource for attracting users. (2) Kuaishou app and viewer. As a content platform, providing content with good quality is the most fundamental business for Kuaishou. With big data technology, Kuaishou can recommend short video to viewers based on their interest and location. As for the viewers, they enjoy the video content on Kuaishou and give rewards for the content that they like. The most important contribution of viewers is 
their feedback on the content and the platform interactions such as "like", "dislike" "retweet", and "share". These feedback items represent a source of big data for Kuaishou, allowing Kuaishou to improve and develop the algorithm's service and recommendation logic. Further, all the feedback energizes and reinforces the behaviors, attitudes, and signifiers that viewers and live-streamers enjoy, forming a culture associated with Kuaishou. (3) Kuaishou app and third parties such as offline shops, factories, and advertisers. Regarding third parties, Kuaishou provides them with low-cost channel for promotion and marketing. The large user base of Kuaishou is quite attractive for third parties who want easy and low-cost access to customers to gain exposure for their brand and product. Regarding Kuaishou, offline shops, factories, and advertisers have the same, role though with various mechanisms. Offline shops/factories co-operate with the MCN and its content creators/live-streamers to promote their product in the form of short videos or live-streaming. Advertisers find a marketing platform to make and promote commercials on the Kuaishou app in the form of short videos. In return, Kuaishou gathers commissions and service fees from third parties. Thus, we conceptualize a simplified, overall business model of the Kuaishou platform in Figure 2 below.

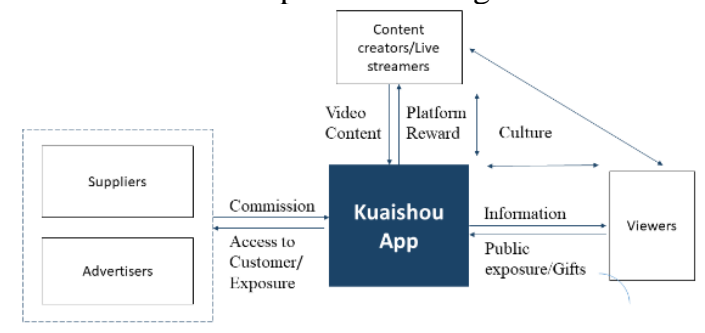

\section{Figure 2. Business Model of Kuaishou}

4.1.1. Value constellation model of Kuaishou. Next we discuss the three gravities model. Applying the model to a content platform, we can analyze the value co-creation mechanism of the value constellation in more detail. When considering the gravities among Kuaishou, content creators, viewers and third parties, the value co-creation process can be divided into two: paths including Kuaishou (internal) and paths excluding Kuaishou (external path).

Internal gravity refers to the interaction force among Kuaishou and four other players including viewers, content creators, and advertisers with suppliers. Each of the three relationships and the ways they interact with the app are presented in detail below and then summarized in Table 1.
4.1.2. Viewers - Kuaishou. Much of the internal attraction between viewers and Kuaishou stems from the functions embedded in the Kuaishou app.

Same City: Viewers can discover short videos made by other users in the same city through the "Same city" page. Kuaishou provides a chat function for them to make private communications. Same City also makes it possible for those viewers who have the same interest and live in the same city to have a group chat and even meet offline, which expands the network of viewers.

Viewer recommendation mechanism: On Kuaishou, each video has tags that generate metadata. Kuaishou recommends short videos based on the user's preferences derived from the frequency of clicks on the tag and the average viewing time of short videos. Every viewer has their own sets of short videos and every short video can find appreciators.

Group Chat: As a short video social app, Kuaishou also has social functions including group chat. These group chats are established for various domains, such as hobbies, location, fans of the same content creator, and so on.

My Page: Here, users can manage the basic information of their account including their followers and content creators. Also, they can post and manage their own works, creating their online space.

User Interface: At the top of the home page, are three buttons, Following, Discovery, and Selected. On the Following page and Discovery page, short videos are presented in waterfall style, that is, there are many short videos on one page, and the viewer can scroll up and down to select videos to watch. However, the Selected page delivers full screen presentation providing more immersive experiences.

Interaction: Viewer interaction on the app includes buttons for like, comment, follow, share, and shopping. Viewers can also send comments to the live-streamer in real-time, which strengthens the relationship between the viewers and the livestreamer, while giving immediate feedback on the content presented.

Lao-tie relationship: This is a non-electronic function preferred and promoted throughout the platform. Kuaishou is committed to establishing a lao-tie relationship between the content creators and the viewers. Kuaishou encourages content creators to call their followers lao-tie in short videos, the very basis of culture in the app. Lao-tie attitude indicates a casual "hey-buddy" relationship - equal, and personal. It is folksy, but widely accepted.

4.1.3. Content creators - Kuaishou. Appreciation gifts function: The content-creators can earn revenue from the number of plays of short videos as well as 
the appreciation gifts from the viewers. As previously mentioned, the viewers give e-stickers as signs of appreciation to the content-creators in the field of live-streaming. Usually, Kuaishou platform takes about $50 \%$ of the total value and pays the remaining $50 \%$ to the live-streamers. This procedure transforms the content creations into financial benefits.

Short Video Making: This is a basic function of Kuaishou, with which content-creators can freely create short videos from 15 seconds up to 5 minutes with a variety of embedded tools. This function lowers the barriers to video production and gives everyone the opportunity to create value from their inspiration and daily life.

Live-streaming: Real-time bullet comments during live-streaming, giving appreciation, and other direct interactions bring the content-creators and the viewers closer. The communication between these two players helps to improve the content of short videos.

Sales Representative: Kuaishou has become a popular marketing platform because of the low cost of live-streaming, the large user base and the low technical threshold. Some offline suppliers have their own live-streaming to sell products, some look for suitable and influential live-streamers as sales representatives to sell their products.

Recommendation mechanism (for content creators): Kuaishou recommends short videos according to two indicators: clout and time of release. New videos have a high level of clout, and over time their exposure decreases. Videos that were extremely popular will also lose exposure after reaching a certain clout threshold. This mechanism gives common content-creators more opportunity to show their work to the viewers.

\subsubsection{Advertisers and suppliers - Kuaishou.} Marketing Platform: Advertisers can hand over promotion materials to this marketing platform. The platform creates the commercials and puts them on Kuaishou as requested.

Promotion Video: Advertisers can also create their own short promotion videos and pay a service fee to put them on Kuaishou, placed in a fixed position on each page.

Live Shopping: Viewers can click on the shopping button to purchase the recommended products directly while watching live-streaming.

Live Shopping Festival: Kuaishou holds a liveshopping festival every year based on live-streaming.

My Shop: Besides using standard e-commerce platforms, suppliers can also open their own online shops on Kuaishou.
Live-streaming Base: In order to manage the quality of the goods and improve the professional skills of the live-streamers, Kuaishou has established bases around China which are used to select products and match suitable live-streamers to sell goods via live-streaming. Suppliers can receive professional training as live-streamers at the bases.

Product Sourcing: In order to give offline suppliers more opportunity to participate in livestreaming, Kuaishou cooperates in collecting products and shares logistics management experience to help maintain quality and low price.

The Kuaishou business model described above illuminates its ecosystem. Kuaishou, the hub, provides value by creating offerings from training to quality control. Viewers and content makers meet through the app while third parties, suppliers and other intermediaries take orders and build relationships. These participants can dynamically support, overlap, and replace each other in the ecosystem.

\section{Discussion}

Table 1 below summarizes the functions described in the previous section of and relates them to the internal gravity to which they contribute.

Table 1. Platform participants' use of value gravities

\begin{tabular}{|c|c|c|c|c|}
\hline $\begin{array}{l}\text { Partici } \\
\text { pant } \\
\text { pair }\end{array}$ & $\begin{array}{l}\text { Internal } \\
\text { gravity } \\
\text { source }\end{array}$ & $\begin{array}{l}\text { Value- } \\
\text { recogni } \\
\text { zing }\end{array}$ & $\begin{array}{l}\text { Valu } \\
\text { e- } \\
\text { shari } \\
\text { ng }\end{array}$ & $\begin{array}{l}\text { Value } \\
- \\
\text { realizi } \\
\text { ng }\end{array}$ \\
\hline \multirow{7}{*}{ 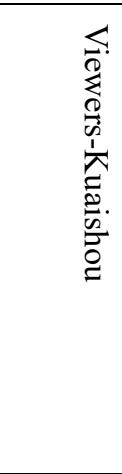 } & Same city & Yes & Yes & No \\
\hline & $\begin{array}{l}\text { Recommen } \\
\text { dation } \\
\text { Mechanism }\end{array}$ & Yes & Yes & No \\
\hline & Group Chat & Yes & Yes & No \\
\hline & My page & No & Yes & No \\
\hline & $\begin{array}{l}\text { UI (image) } \\
\text { design }\end{array}$ & Yes & No & No \\
\hline & Interaction & Yes & Yes & No \\
\hline & $\begin{array}{l}\text { Lao-tie } \\
\text { relationship }\end{array}$ & Yes & No & No \\
\hline \multirow{4}{*}{ 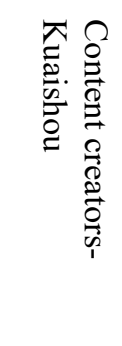 } & $\begin{array}{l}\text { Appreciatio } \\
\mathrm{n}\end{array}$ & No & Yes & Yes \\
\hline & $\begin{array}{l}\text { Short Video } \\
\text { Making }\end{array}$ & Yes & Yes & Yes \\
\hline & $\begin{array}{l}\text { Live } \\
\text { streaming }\end{array}$ & Yes & Yes & Yes \\
\hline & $\begin{array}{l}\text { Sales } \\
\text { Represent. }\end{array}$ & No & Yes & Yes \\
\hline
\end{tabular}




\begin{tabular}{|c|c|c|c|c|}
\hline & $\begin{array}{l}\text { Recommen } \\
\text { dation } \\
\text { Mechanism }\end{array}$ & Yes & Yes & No \\
\hline \multirow{7}{*}{ 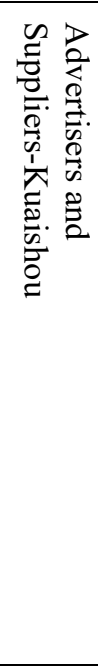 } & $\begin{array}{l}\text { Marketing } \\
\text { Platform }\end{array}$ & Yes & Yes & Yes \\
\hline & $\begin{array}{l}\text { Promotion } \\
\text { Video }\end{array}$ & Yes & Yes & Yes \\
\hline & $\begin{array}{l}\text { Live } \\
\text { Shopping }\end{array}$ & No & Yes & Yes \\
\hline & $\begin{array}{l}\text { Live } \\
\text { Shopping } \\
\text { Festival }\end{array}$ & Yes & Yes & Yes \\
\hline & $\begin{array}{l}\text { My Own } \\
\text { Shop }\end{array}$ & No & Yes & Yes \\
\hline & $\begin{array}{l}\text { Live- } \\
\text { streaming } \\
\text { Base }\end{array}$ & Yes & Yes & Yes \\
\hline & $\begin{array}{l}\text { Product } \\
\text { sourcing }\end{array}$ & Yes & Yes & Yes \\
\hline
\end{tabular}

The table above emphasizes that in each of the three relationships, all three gravities are present and help the players work together to create value. However, we can see that in each relationship, there is a more influential one which dominates the development of the relationship.

\subsection{Internal gravities}

In the viewer-Kuaishou relationship, the valuerecognizing gravity is the main gravity that sustains the value-creation process. The viewer's preference for short video content creates a unique style and cultural atmosphere which becomes the cultural image of Kuaishou and attracts the viewers with similar tastes and interests. Through the social function of Kuaishou, more viewers with the same interests and tastes come together to form social communities through Kuaishou. Kuaishou promotes folksy and casual lao-tie culture which appeals to the user base from second and third tier cities in China. Culture appears as the strongest aspect of this gravity: though it is notably not clickable in the interface, lao-tie culture permeates the platform as a soft component. Within this value-recognizing gravity, viewers no longer simply consume value, but each of their small behaviors provides direction and feeling and attitude, that is to say culture, for the future creation of short videos on the platform as a whole. The lao-tie relationship exists also between viewers and content makers. This culture has the function of delivering a flow of viewers and live streamers into the app [32]. In other MCNs, this relationship does not exist and therefore they have less participation among the target market of lesser cities and rural areas which drive user participation in the app. In short, lao-tie culture appears to maintain user numbers, flow of new entrants, and user engagement. The app boosts the culture and the culture boosts the app. The result is a competitive advantage in gaining and keeping participants in the platform. The advantage is clearly on display inside China, however precisely the finely tuned strength of that culture may be a cause of weak results internationally where Kuaishou has struggled to gain traction. Vahlne and Bhatti [33] showed that processes of relationship commitment can be microfoundations contributing to internationalization, however in the case of Kuaishou, those processes, which are very strong in China, may not exist for the platform in international settings.

In the relationship between Kuaishou and content-creators, the value-realizing gravity plays the greatest role among the three gravities. Although content-creators start creating short videos for the purpose of sharing their life and inspirations, the business mechanism of the Kuaishou allows such creations to become a professional and permanent job choice. A short video will be recommended according to a certain recommendation mechanism, and will get certain clout according to the number of comments, likes and retweets. At the same time, content-creators can also produce short videos for other businesses to earn advertising fees. It can be considered that the realization mechanism established by Kuaishou for content-creators attracts many talented creators and also motivates them to produce high quality videos. MCNs, as the agencies for content-creators, have become the bridge between Kuaishou and content-creators. On the one hand, MCNs can provide professional short video-making guidance to content-creators, and on the other hand, they can also receive and manage commercial orders allowing content-creators to earn more income. Value-realizing gravity also plays an important role in the relationship between the third parties and Kuaishou. Offline suppliers, especially small shops, are lacking cost-efficient promotion channels. On Kuaishou, however, simply by making a few short videos introducing the products or live-streaming the production operations, orders from all over China come in. This is the exact kind of promotion that offline shops with high quality products but no promotion channels and few resources need. These shops benefit from Kuaishou's support to the livestreaming business, especially from live-streaming bases established to offer big data and technical support. Suppliers can also get live-streaming training and quality control management at the source 
of the goods to ensure product quality and transport efficiency. Small offline suppliers are attracted by the convenience of short videos and live-streaming as a marketing tool, which makes live-streaming the fastest growing business unit of Kuaishou.

The gravities directly relating Kuaishou to the players, internal gravities, are depicted in Figure 3.

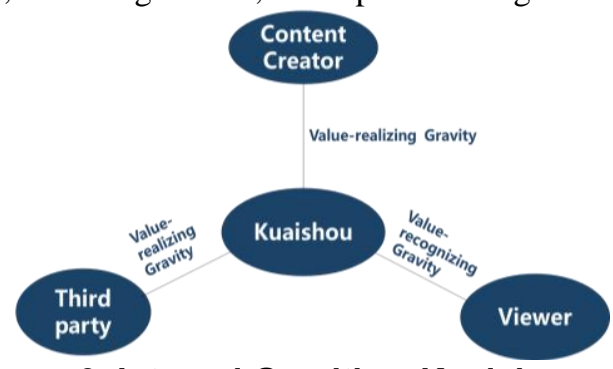

Figure 3. Internal Gravities, Kuaishou

The internal gravities function in the relationship of Kuaishou with other players. But in real business activities, viewers, content-creators and the third parties are also attracted to each other in the value constellation. Externally of the focal firm, it is the value-sharing gravity that connects them. These external gravities are discussed next.

\subsection{External gravities}

On Kuaishou, the viewers generate big data by interacting with the short videos, while the value created by the content-creator is short videos and live-streaming. Every like, retweet and comment from the viewer is recorded as data by Kuaishou. Kuaishou then sets the benchmark and direction for the content-creators according to the data. As a result, the viewers are entertained and their behaviors are also transformed into data for the content-creators, allowing them to understand the needs of their viewers and create videos more effectively. This flow of information creates a positive reinforcement feedback loop that strengthens the Kuaishou platform. At the same time, viewers can also show appreciation to their favorite content-creators and live-streamers thereby establishing a relatively equal and intimate relationship with them. As for the content-creators, they can turn their inspirations into works of art and gain the love and financial benefits from the viewers as well as gain self-realization. Some live streamers have been extraordinarily successful, among these is Xinba who grossed over $\$ 300$ million in sales in a 12-hour period [34]. Pop star Jay Chou earned over $\$ 3.4$ million in a 30minute session [35]. Xin Youzhi, known as Peasant CEO, was able to spend $\$ 7$ million on a wedding and make major charitable gifts while others have launched careers in sales, music, performance and so on [16], [36]. During the process, both contentcreators and viewers create value together, constantly contributing to the improvement of the quality of short videos and satisfying the needs of each side.

The mechanism of the value sharing gravity between content-creators and third parties is totally different than the relationships. In this relationship, the content-creators are usually acting as sale representatives. On Kuaishou, many third parties are not large companies, but small offline shops and suppliers. They create products with high quality but lack online promotional channels to expand the popularity of their products. In the meantime, content-creators with certain public exposure also need a suitable realization mechanism to support their career choice. Therefore, the marketing need of offline suppliers and the financial need of contentcreators match perfectly, giving rise to the business of selling live-streaming. As a result, the third party pays the content-creators agreed commissions, and the content-creators create short videos for the products, recommending them to followers who buy the products to show their love, trust and support to the content-creator. The third party gains public exposure as well as revenue boosts. Of particular interest is that the followers are not annoyed by such promotional short videos, because they enjoy the content innovation, and some promotional short videos are even more interesting than ordinary ones. This cycle of interactions throughout the Kuaishou ecosystem creates a positive feedback loop that reinforces value for third parties, viewers, and live streamers. This value-sharing gravity stimulates the creativity that the participants bring to the platform.

Mainly, third parties sell low value items on Kuaishou. Viewers know about these via short videos and live-streaming. When viewers give feedback, they allow suppliers to improve the quality of their products and services accurately. Value-sharing gravity makes the products and services created by third parties available to a wider range of customers, thereby improving efficiency of resource allocation.

The external relationships are shown in Figure 4 with benefits the players gain from the relationships. 


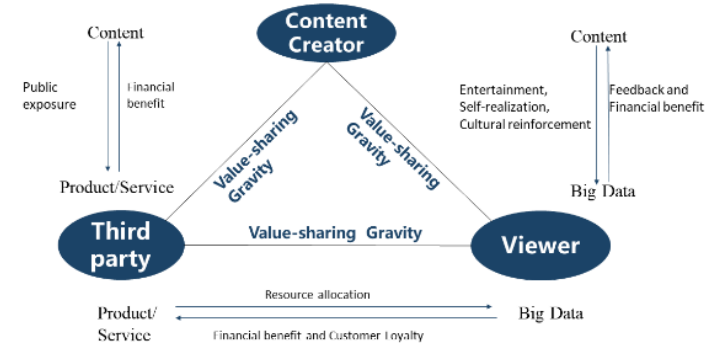

Figure 4. External Gravities, Kuaishou

\section{Conclusion}

Applying Luo's gravities theory, this report discusses the value co-creation mechanism with value constellation via a case study of Kuaishou. Luo argues that value constellation is a gravity aggregation formed by manufacturer, supplier, partner, distributor and customer. The gravities consist of value-recognizing gravity, value-realizing gravity, and value-sharing gravity.

In the value constellation of Kuaishou, the main players are Kuaishou, the viewers, the contentcreators and the third parties (consisting of advertisers and suppliers). These players are brought together by three value gravities as they co-create value. Economic interest and the app's lao-tie culture are key mechanisms that motivate and draw them into the platform. This study finds these three gravities are divisible into internal and external gravities based on the relationship of players.

Internal value gravity maintains the relationship between Kuaishou and each of the other players. In the relationship between the viewers and Kuaishou, it is primarily the value-recognizing gravity that brings the two players together. In the relationship between the content-creators and Kuaishou, it is mainly the value-realizing gravity at work. Between the third parties and Kuaishou, value-realizing gravity also dominates value co-creation.

Among the external relationships, it is mainly the value-sharing gravity that works between the player dyads. Between the content-creators and the viewers, the content-creators create short videos and livestreaming to provide entertainment value to the viewers as well as gain a sense of self-realization. The viewers enjoy the leisure time, provide financial benefits to their favorite content-creators, and also provide feedback in form of big data, setting benchmarks and directions for content creation. In the content-creator and the third party relationship, the content-creators produce promotion short videos for products and services created by the third party, helping the third party to gain public exposure and build brand awareness. The third party pays the creator commissions, which improve the sustainability of the creator's work. In the relationship between third parties and the viewers, the viewers watch promotional videos and livestreaming to know and purchase products, giving financial benefits to the third party. And as viewers give more feedback on the experience of the products, the third party makers can improve the products and services to earn positive, digital wordof-mouth reputations. Figure 5 shows the whole interactive, dynamic value constellation.

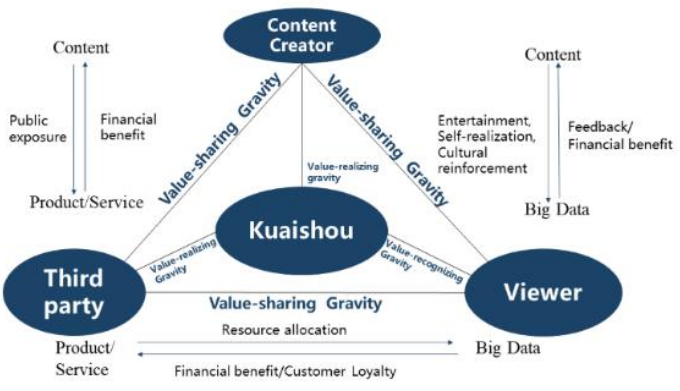

Figure 5. Kuaishou Value Constellation

The platform is the centerpiece of the gravities and allows the other parts of the constellation to remain in balance by cycling information, revenue, data, knowledge about appropriate creativity, content, and services through the ecosystem. As a Born Digital organization, Kuaishou was able to build the culture of its target audience into its interactions and success. The platform's folksy lao-tie culture especially allows reinforcing feedback loops that support dynamic information flows and thus users' value co-creation. Because the appearance and movement of knowledge is dynamic, the gravities are able to compensate and adjust via the robust presence of the Kuaishou app. Regarding its still-nascent, Gradually Global nature, Kuaishou's lao-tie culture, an important part of its value creation mechanisms, may need to transform or be shed in order to develop strong followings in other countries. Other firms and platforms can learn to gain benefits and avoid pitfalls from Kuaishou's exploitation of culture.

Academics may wish to extend Luo's gravities theory to non-digital, conventional firms and ecosystems as this may provide fruitful insights into how members interact and impact value creation.

This study's limitations include that reporting on creators and users in depth was beyond the current scope. Further, it was not possible to get insights directly from company executives, although media revealed some information. 


\section{References}

[1] M. Luo, "Value Constellation: Theoretical Explanation and Construction of Value-creating Mechanism; 价值 星系: 理论解释与价值创造机制的构建, ” China Ind. Econ., vol. 214, no. 1, pp. 80-89, 2006.

[2] S. L. Vargo and R. F. Lusch, "Service-dominant logic: Continuing the evolution,” J. Acad. Mark. Sci., vol. 36, no. 1, pp. 1-10, 2008.

[3] A. Hein et al., "Digital platform ecosystems," Electron. Mark., vol. 30, no. 1, pp. 87-98, 2020.

[4] S. Monaghan, E. Tippmann, and N. Coviello, "Born digitals: Thoughts on their internationalization and a research agenda," J. Int. Bus. Stud., vol. 51, no. 1, pp. 11-22, 2020, doi: 10.1057/s41267-019-00290-0.

[5] A. Gawer, "Platforms, markets and innovation: an introduction," in Platforms, Markets and Innovation, A. Gawer, Ed. Cheltenham, UK: Elgar, 2009, pp. 1-16.

[6] A. Ojala and K. Lyytinen, "Competition Logics during Digital Platform Evolution," in Proceedings of the 51st Hawaii International Conference on System Sciences (HICSS 2018), 2018, no. HICSS, pp. 1026-1035.

[7] R. Ramírez, "Value co-production: Intellectual origins and implications for practice and research," Strateg. Manag. J., vol. 20, no. 1, pp. 49-65, 1999.

[8] S. L. Vargo, R. F. Lusch, and M. A. Akaka, "Advancing Service Science with Service-Dominant Logic: Clarifications and Conceptual Development," in Handbook of Service Science, Service Science: Research and Innovations in the Service Economy, P. P. Maglio, et al. C. A. Kieliszewski, and J. C. Spohrer, Eds. New York, NY: Springer Science \& Business Media, 2010, pp. 133-156.

[9] M. Fransman, Innovation Ecosystems: Increasing Competitiveness. Cambridge, UK: Cambridge University Press, 2018.

[10] E. Anggraeni, E. Den Hartigh, and M. Zegveld, "Business ecosystem as a perspective for studying the relations between firms and their business networks," in Eccon 2007, 2007, pp. 1-28.

[11] G. T. Gundlach, "Complexity Science and Antitrust?," Antitrust Bull., vol. 51, no. 1, pp. 17-30, 2006.

[12] Y. Xuecheng and T. Xiaobo, "From physical value chain, value matrix to flexible value network: example of Xiaomi Corporation's social value co-creation," Manage. Rev., vol. 27, no. 7, pp. 232-239, 2015.

[13] C. Li, H. Chen, and J. Zhang, “第三方买单\&商业模式 价值共创内在 123,” J. Guangxi Univ. Financ. Econ., vol. 33, no. 1, pp. 117-127, 2020.

[14] J. Wang and D. Sun, “价值共创视角下短视频平台商 业模式研究——基于抖音短视频的案例研究), ”出 版发行研究, vol. 18, pp. 20-26, 2018.

[15] J. Lin and J. de Kloet, "Platformization of the Unlikely Creative Class: Kuaishou and Chinese Digital Cultural Production," Soc. Media Soc., vol. 5, no. 4, 2019.

[16] C. K. K. Tan, J. Wang, S. Wangzhu, J. Xu, and C. Zhu, "The real digital housewives of China's Kuaishou video-sharing and live-streaming app," Media, Cult. Soc., vol. 42, no. 7-8, pp. 1243-1259, 2020.
[17] M. Gabrielsson, S. Fraccastoro, A. Ojala, and M. Rollins, "Digital Entrepreneurial Internationalizers: Definitions, Theoretical Implications, and Research Avenues," in Proceedings of the 54th Hawaii International Conference on System Sciences, 2021, vol. 54, pp. 5069-5078, doi: 10.24251/hicss.2021.617.

[18] M. M. Al-Debei and D. Avison, "Developing A Unified Framework Of The Business Model Concept.," Eur. J. Inf. Syst., vol. 19, no. 3, pp. 359-376, 2010.

[19] C. Grönroos and J. Gummerus, "The service revolution and its marketing implications: service logic vs servicedominant logic," Manag. Serv. Qual., vol. 24, no. 3, pp. 206-229, 2014, doi: 10.1108/MSQ-03-2014-0042.

[20] M. E. Porter, "Technology and competitive advantage," J. Bus. Strategy, vol. 5, no. 3, pp. 60-78, 1985.

[21] M. Zhou and S.-D. Liu, "Becoming precarious playbour: Chinese migrant youth on the Kuaishou video-sharing platform," Econ. Labour Relations Rev., p. $103530462110370,2021$.

[22] M. B. Miles, A. M. Huberman, and J. Saldaña, Qualitative Data Analysis: A Methods Sourcebook, 4th ed. Thousand Oaks, CA: Sage Publications, 2018.

[23] Kuaishou, "Milestones," 2021. https://www.kuaishou.com (accessed May 17, 2021).

[24] T. Qu, "Kuaishou joins 1 billion user club on the back of live streaming in China, international expansion," South China Morning Post, Jun. 23, 2021.

[25] Z. Huang, "Billionaire Who Missed Out on TikTok," Bloomberg.com, 2021. https://www.bloomberg.com/news/articles/2021-0721/billionaire-who-missed-out-on-tiktok-is-nowtrying-to-beat-it (accessed Aug. 26, 2021).

[26] P. Zhang, "How Kuaishou achieved the goal of 300 million daily active users," CnTechPost, Beijing, pp. 18, Feb. 2020.

[27] "Kuaishou's shares surge on the video app's stockmarket debut," The Economist, Feb. 2021.

[28] D. Liu, “快手出海不利,”财经天下, no. 7, pp. 38-40, 2019, [Online]. Available: http://www.ixueshu.com.

[29] L. Matsakis, "Zynn, the Hot New Video App, Is Full of Stolen Content," Wired, pp. 1-9, Jun. 2020.

[30] R. McMorrow, "Kuaishou IPO boosts biggest rival to China's TikTok," Fin. Times, Beijing, Feb. 01, 2021.

[31] A. Tiwana, Platform Ecosystems: Aligning Architecture, Governance, and Strategy. Amsterdam: Elsevier Science, 2014.

[32] M. Li, C. K. K. Tan, and Y. Yang, "Shehui Ren: cultural production and rural youths' use of the Kuaishou video-sharing app in Eastern China," Inf. Commun. Soc., vol. 23, no. 10, pp. 1499-1514, 2020.

[33] J. E. Vahlne and W. A. Bhatti, "Relationship Development: A Micro-Foundation for the Internationalization Process of the Multinational Business Enterprise," Manag. Int. Rev., vol. 59, no. 2, pp. 203-228, 2019.

[34] I. Deng, "Comeback king," South China Morning Post, Mar. 28, 2021.

[35] T. Tan, "Jay Chou's First Live Stream On Chinese App Kuaishou," 8 Days, Singapore, Jul. 2020.

[36] L. Wang, "Life in Kuaishou's Watery Margins," The Sixth Tone, Shanghai, 2020. 